\title{
Immunocytochemical Localization of the Erythroid Glucose Transporter: Abundance in Tissues with Barrier Functions
}

\author{
Sami I. Harik, ${ }^{1}$ Rajesh N. Kalaria, ${ }^{1}$ Lars Andersson, ${ }^{3}$ Per Lundahl, ${ }^{3}$ and George Perry ${ }^{2}$ \\ Departments of ${ }^{1}$ Neurology and ${ }^{2}$ Pathology, University Hospitals of Cleveland and Case Western Reserve University \\ School of Medicine, Cleveland, Ohio 44106 and ${ }^{3}$ Department of Biochemistry, University of Uppsala, Uppsala, Sweden.
}

\begin{abstract}
We investigated the cellular localization and tissue distribution of the glucose transporter protein in the nervous system of the monkey and rat, and in other tissues of the rat, by immunocytochemical methods with monoclonal and polyclonal antibodies to the glucose transporter of human erythrocytes. We found intense immunostaining, indicating a high density of the glucose transporter, in all intraparenchymal blood vessels of the brain and spinal cord, in pial vessels, and in endoneurial capillaries of peripheral nerves, nerve roots, and dorsal root ganglia. Larger blood vessels at the base of the brain and in major fissures did not stain. The only intraparenchymal brain microvessels that did not immunostain were in circumventricular organs. There was no specific immunostaining of neurons or glia, except for tanycytes in the floor of the third ventricle, which immunostained intensely. Vessels of the choroid plexus did not stain, but the choroid epithelium, especially its basal membranes, stained. The only non-neural organ where immunostaining was evident in its microvessels was the testis. In addition to the endothelium of neural and testicular tissues, there was immunostaining in certain epithelial tissues such as the perineurium of peripheral nerves and nerve roots, the epithelium of the ascending loop of Henle in the kidney, and the epidermis of the skin. Based on these findings, we hypothesize that a high density of the erythroid-type glucose transporter is inherent to many endothelial and epithelial cells that are joined by occluding junctions. However, other epithelial tissues with known occluding intercellular junctions that lack the erythroid-type of glucose transporter may have other types of glucose transporter proteins.
\end{abstract}

Facilitative glucose transporter (GT) proteins are ubiquitous in cell membranes of tissues that use glucose, where they play a crucial role in transporting the water-soluble D-glucose across the lipid bilayer of plasma membranes (see reviews by Bell et al., 1990; Kasanicki and Pilch, 1990; Thorens et al., 1990). Of the 4 or 5 members of the GT family that are thus far known,

Received May 10, 1990; revised Aug. 16, 1990; accepted Aug. 17, 1990

This work was supported by U.S. Public Health Service Grants HL-35617 and AG-00415 and by the David S. Ingalls Fund. Production of the monoclonal antibody was supported by the Swedish Natural Science Research Council. We wish to thank Dr. Steven Emancipator for helpful discussions, Dr. Ora M. Rosen for donation of the GT antiscra, and Dr. S. Ledbetter for donation of collagen type IV antiserum. We also thank Paul M. Whitney, Fares Abdallah, Peggy Richey, Sandra Siedlak, and Paul Mulvihill for technical assistance and Jeanette Barnhart for manuscript preparation.

Correspondence should be addressed to Dr. Sami I. Harik, Department of Neurology, University Hospitals of Cleveland, Cleveland, OH 44106.

Copyright @ 1990 Society for Neuroscience $0270-6474 / 90 / 123862-11 \$ 03.00 / 0$ the human erythrocyte GT is most extensively studied, and it is now accepted that it mediates glucose diffusion in a manner that is saturable, stereospecific, nonconcentrative, nonenergy requiring, and not influenced by sodium or insulin.

We previously demonstrated that the density of GT in isolated rat and pig brain microvessels, assessed by the D-glucose-displaceable specific binding of cytochalasin $\mathrm{B}$, was second only to that of human erythrocyte membranes and about 10-fold higher than in brain homogenates (Dick et al., 1984; Dick and Harik, 1986). Membrane-enriched subcellular fractions of brain microvessels had an even higher density of GT (maximal binding of cytochalasin B exceeded $90 \mathrm{pmol} / \mathrm{mg}$ protein; Dick et al., 1984). Irreversible photoaffinity labeling of cytochalasin B to brain microvessels, followed by SDS-PAGE, allowed estimation of the $M_{r}$ of brain microvessel GT at about $53 \mathrm{kDa}$, similar to that of human erythrocytes (Dick et al., 1984). Furthermore, antiserum to purified GT of human erythrocytes reacted with a polypeptide in a band corresponding to an $M_{r}$ of about $53 \mathrm{kDa}$ on immunoblots of brain microvessel preparations, indicating similar antigenic properties (Dick et al., 1984). Subsequent work confirmed and extended these findings to sheep (Baldwin et al., 1985), cows (Kasanicki et al., 1987), and humans (Kalaria et al., 1988; Kalaria and Harik, 1989). The availability of molecular probes for GTs allowed studies of their mRNA tissue distribution. Northern blots revealed high levels of the erythroid-type GT mRNA in brain microvessels (Flier et al., 1987; Weiler-Güttler et al., 1989), and consistent findings were obtained by in situ hybridization (Kalaria et al., 1989). The recent availability of antibodies to the human erythrocyte GT and to other GT types permits their precise localization and tissue distribution, which is not yet known, but which was surmised from mRNA blotting or in situ hybridization studies. Immunocytochemical studics documented the presence of a high density of the erythroid-type GT in human (Kalaria et al., 1988) and dog (Gerhart et al., 1989) brain microvessels and peripheral nerve perineurium (Froehner et al., 1988) and in certain endothelial and epithelial cells of the eye (Harik et al., 1990) with exquisite anatomical detail.

About 25 yr ago, Crone predicted the existence of a GT in endothelial cells of brain capillaries to allow the entry of glucose from the blood to the brain (Crone, 1961, 1963, 1965). His opinions met with strong resistance because of the prevalent notion that glial processes formed the anatomical basis of the blood-brain barrier (BBB), and because few scientists then believed that the tiny endothelial cells of brain capillaries were capable of undertaking so vital a task. In retrospect, the existence of a high density of the GT in brain endothelial cells is logical in view of the high brain metabolic rate for glucose, the isolation 


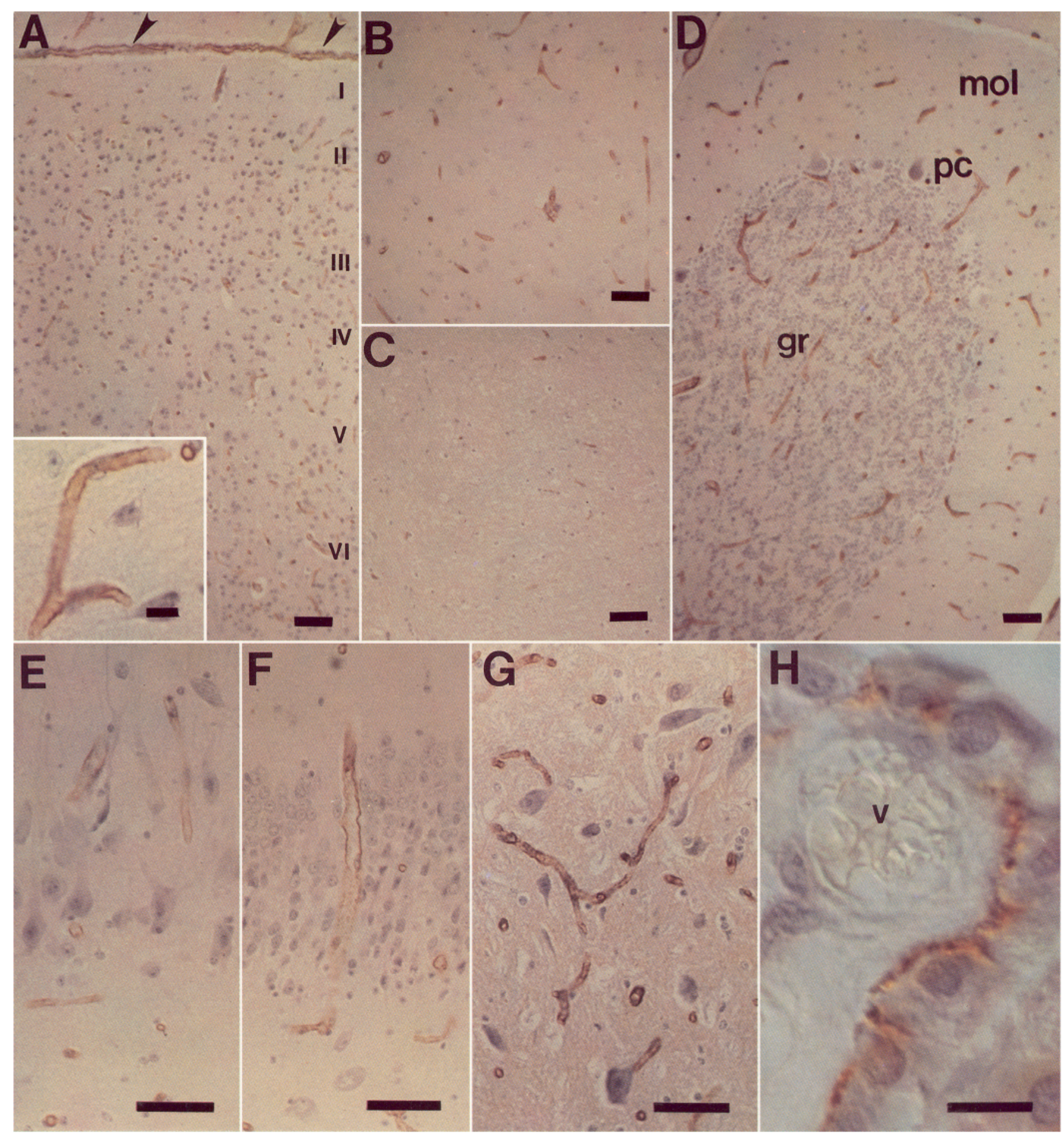

Figure 1. Immunocytochemically stained sections of monkey brain using monoclonal antibody to erythroid GT, later counterstained with hematoxylin. A, A section of the monkey occipital cerebral cortex. Roman numerals indicate the cortical layers. Pial vessels (arrowheads) were stained. Note the discrepancy in microvessel density among the cortical layers, which is highest in layers III and IV and lowest in layer I. The inset depicts a high-power view of a cortical microvessel. $B$, A section of the caudate nucleus, a subcortical gray matter region of the brain. $C$, A section of the internal capsule, a white matter region. Note the low vessel density in the white matter in comparison to that of gray matter regions depicted in $A$ and $B . D$, A section through the cerebellum with its molecular $(m o l)$, Purkinje cell $(p c)$ and granular layers $(g r)$. There is an apparent lower density of microvessels in the molecular layer. $E$, A higher-power view through Sommer's sector of the hippocampus. $F$, A similar view of the dentate gyrus. $G$, The nucleus locus coeruleus of the pons, with its dense vascularity. $H$, A high-power view of the choroid plexus showing the brown reaction product mostly in the basal membranes of the choroid epithelium, but none in blood vessels (v) of the choroid. $A-G$ were viewed under bright-field illumination, and $H$ was viewed under differential interference contrast (Nomarski) illumination. Scale bars: $A-G, 50 \mu \mathrm{m}$; inset in $A$ and $H, 10 \mu \mathrm{m}$. 


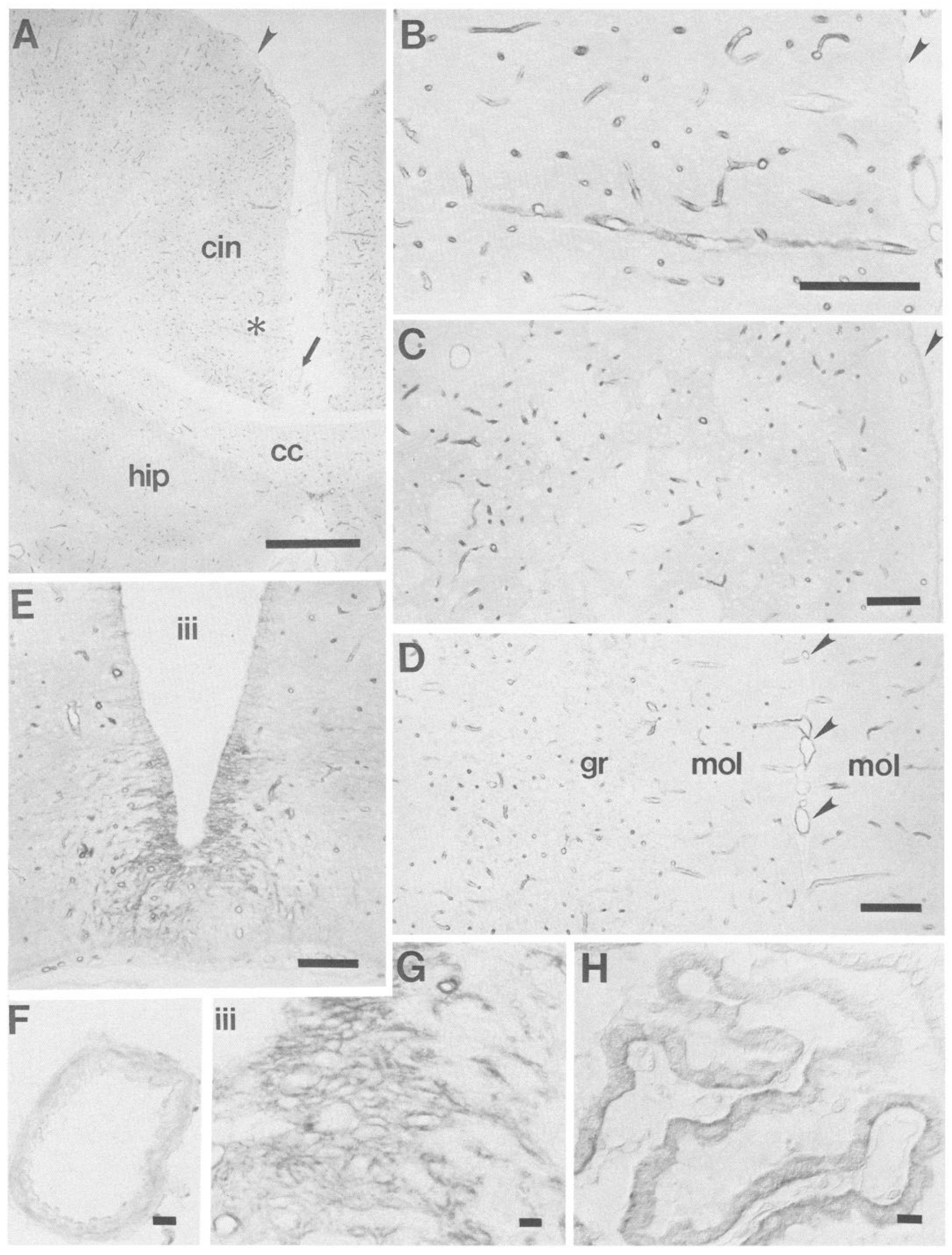


of the brain behind capillaries with occluding interendothelial junctions (i.e., the BBB), and the fact that brain capillaries, which constitute less than $1 \%$ of the brain weight, must transport glucose for the much larger mass of surrounding neurons and glia.

The object of this work was to determine by immunocytochemical methods the precise localization of the erythroid-type GT in the monkey and rat brains and in various tissues of the rat, especially those that are known to possess barrier properties. Our hypothesis was that tissues that do not have barrier properties would allow easy penetration of the water-soluble glucose molecule and therefore should not have a high density of the GT beyond that needed for their own glucose use. However, cells with barrier properties must have a high density of a GT protein to allow glucose entry into the much larger mass of tissue that they encompass. Thus, we reasoned that the presence of a high density of the erythroid-type GT may be a marker of barrier cells. However, barrier cells that do not have a high density of the erythroid-type GT may have a high density of other GT proteins. Thus, intense immunostaining with antibodies to the erythroid GT would be a sufficient but not a necessary marker of cells bearing occluding junctions.

Part of these results were presented in abstract form (Harik et al., 1989).

\section{Materials and Methods}

Tissues. Adult Wistar rats of both sexes were deeply anesthetized with Nembutal and perfused through the heart with saline until the return from the right atrium was clear, then perfusion-fixed with Bouin's solution. Tissues were removed and postfixed in Bouin's fixative for $2 \mathrm{~d}$ prior to dchydration and cmbcdding in paraffin. Tissues were also obtained from adult Green African monkeys immediately after they were killed by barbiturate overdose and fixed by immersion in Bouin's solution for $2 \mathrm{~d}$, then embedded in paraffin.

Antibodies. The primary antibodies that we used to immunostain the GT were monoclonal antibody B315:32, which was directed against the erythroid GT and found by use of synthetic peptides to bind strongly to the C-terminus of this GT (Andersson and Lundahl, 1988), and rabbit antisera to the purified GT of human erythrocytes (Haspel et al., 1985) and to synthetic C-terminus peptide of the erythroid GT (Haspel et al., 1988). Antiserum to collagen type IV (donated by Dr. S. Ledbetter) was used to immunostain the basal lamina of blood vessels. In preliminary experiments, we found that optimal staining was achieved at a $1: 2$ dilution of the monoclonal antibody hybridoma culture supernatant, and at a 1:100 dilution of the antisera. In control incubations, to show the specificity of immunostaining, we used antibodies to neurofilaments or $\tau$ protein as irrelevant antibodies and omitted the primary antibodies, and in the case of the GT antibodies, we adsorbed the primary antibodies with human erythrocyte membrane fragments prior to immunostaining. In all these controls, faint or no specific staining was found. The immunostaining patterns obtained with the mouse monoclonal and the rabbit polyclonal antibodies to the glucose transporter were essentially iden- tical, thus reducing the possibility that the observed staining of a given structure is a cross-reaction artifact.

Immunocytochemical stains. Paraffin sections $(6 \mu \mathrm{m})$ were hydrated, treated for $10 \mathrm{~min}$ with $10 \%$ normal goat serum in Tris-buffered saline $(\mathrm{pH}, 7.6)$, and incubated overnight at $0-4^{\circ} \mathrm{C}$ with 1 of the primary antibodies in buffered saline containing $1 \%$ normal goat serum. Immunocytochemical staining was performed according to the peroxidase-antiperoxidase procedure of Sternberger (1986), with 3',3-diaminobenzidine (Sigma) as cosubstrate. To appreciate histological detail, adjacent sections were stained with hemmatoxylin and eosin. Often, GT-immunostained sections were counterstained with hematoxylin. Double-immunostaining for the GT (monoclonal antibody) and type IV collagen was performed on sections of the rat brain and testis to determine the concordance between the 2 antigens in their microvessels. In the double-immunostains, the second stain employed the alkaline phosphatase-antialkaline phosphatase procedure with fast blue BB (Sigma) as the second chromogen.

\section{Results}

Intense immunostaining in monkey and rat brains with antibodies to the erythroid-type GT was primarily restricted to small blood vessels, particularly capillaries (Figs. 1, 2). The reaction product appeared under high magnification to be in cellular elements within the basal lamina (Figs. 1-3). The endothelium of pial vessels also stained (Figs. $1 A ; 2 A, D$ ), but that of larger blood vessels, such as the arteries at the base of the brain and in the major fissures, did not immunostain (Fig. $2 A, F$ ). The meninges, including the pia, did not stain (Figs. $1 D ; 2 A, D$ ). There was minimal background staining of the neuropil, more in gray than in white matter (Fig. $2 A$ ). Large neurons, such as the Purkinje cells of the cerebellum (Figs. $1 D, 2 D$ ), and neurons of discrete brain-stem nuclei, such as the locus coeruleus (Fig. $1 G$ ), did not immunostain. There was no discernible staining in ependymal cells (Fig. 2C), except those in the floor of the third ventricle overlying the median eminence, where the specialized ependyma, that is, tanycytes, stained strongly (Fig. $2 E$, $G)$. The tanycytes were the only cells of glial origin that stained for the erythroid GT. Blood vessels of the choroid plexus did not stain, but there was distinct staining of the choroidal epithelial cells, especially their basal membranes (Figs. $1 \mathrm{H}, 2 \mathrm{H}$ ).

Virtually all parenchymal microvessels in all regions of the brain, except in circumventricular regions that lack BBB characteristics, stained for the GT. This is based on the results of double-immunostained sections, where all microvessels that were identified by type of IV collagen immunostaining also stained for the GT. Figures 1 and 2 were taken from the monkey and rat cerebral cortex, corpus callosum, internal capsule, striatum, cerebellum, hippocampus, and pons. Microvessel density was higher in gray matter than in white matter (Figs. 1-3). Fur-

\footnotetext{
Figure 2. Immunocytochemical stains of rat brain sections using monoclonal antibody to erythroid GT. $A$, A low-power view of a coronal section of the brain about $6 \mathrm{~mm}$ anterior to the interaural line. The cingulate cortex $(\mathrm{cin})$ is seen overlying the corpus callosum ( $\mathrm{cc}$ ) and the hippocampus (hip). It is apparent that the microvessel density of the cortical gray is higher than that of the subcortical white matter or the corpus callosum. Small pial vessels were stained (arrowhead), but larger vessels such as the branch of the anterior cerebral artery in the interhemispheric fissure (arrow) did not stain. B, A higher-power view of the region of the left cingulate gyrus, which is marked by an asterisk in $A$. Note that there was no staining of the pia (arrowhead), but that all microvessels stained for the GT. $C$, A section through the caudate-putamen showing the ependyma of the lateral ventricle on the extreme right (arrowhead). Note that the ependyma did not immunostain, but that all microvessels stained. $D$, A section through the cerebellum showing the molecular layer $(\mathrm{mol})$, the granular layer $(g r)$, and the intervening Purkinje cell layer. All blood vessels of the cerebellum, including the pial vessels (arrowheads), are stained. There is a lower vascular density in the molecular layer than in the granular layer. $E$. The bottom of the third ventricle ( iii) and the underlying median eminence. Note that the specialized ependyma near the median eminence (tanycytes) is stained, whereas the ependyma in the dorsal part of the third ventricle did not stain for the GT. $F$, A high-power view of the interhemispheric artery that is marked by an arrow in $A$. The endothelium of this artery did not stain for the GT. $G$, A high-power view of the tanycytes of the ventral portion of the third ventricle (iii). $H$, A high-power view of the choroid plexus showing immunostaining in the choroid epithelium, especially in its basal membranes. There is no staining of the choroid blood vessels. $A-G$ were viewed under bright-field illumination, and $H$ was viewed under differential interference contrast (Nomarski) illumination. Scale bars: $A, 500 \mu \mathrm{m} ; B-E, 100 \mu \mathrm{m} ; F-H, 10 \mu \mathrm{m}$.
} 

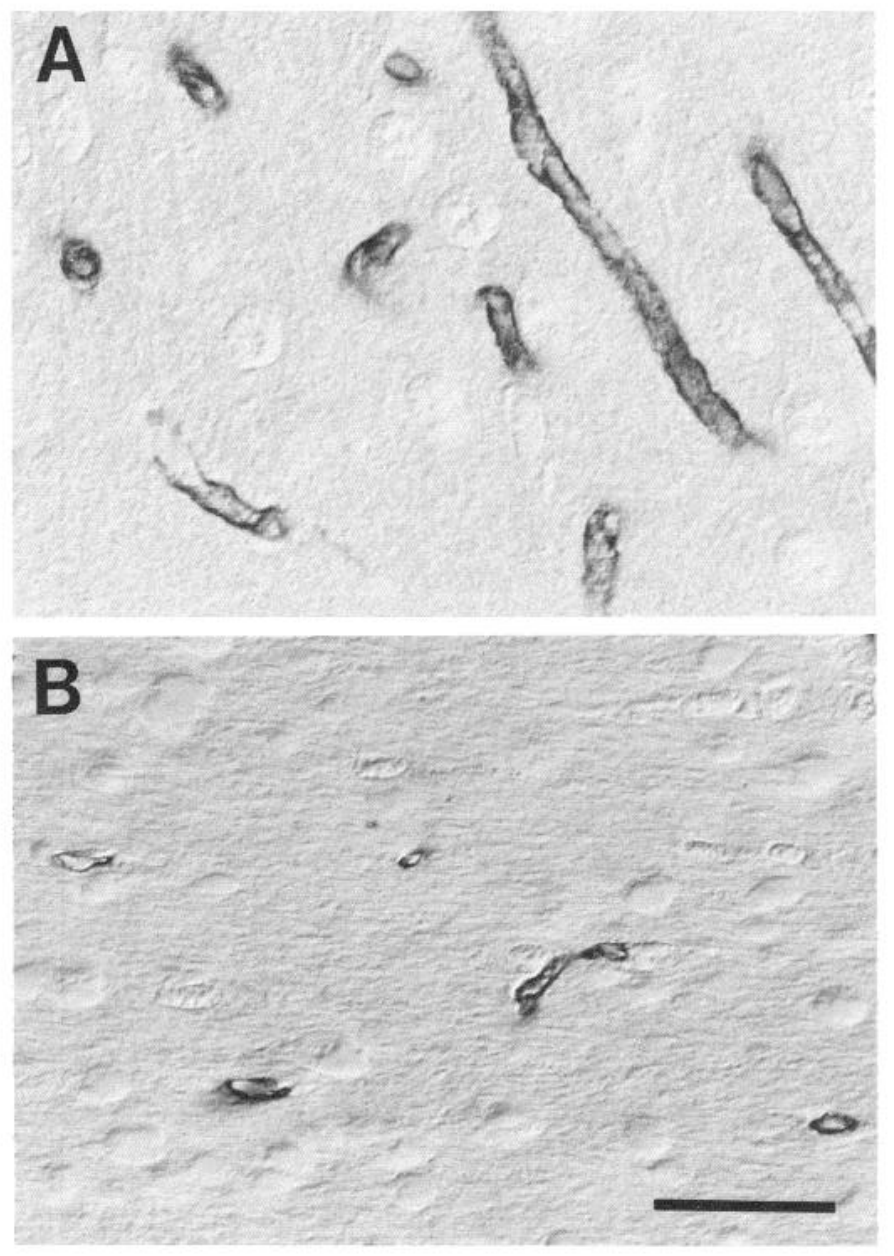

Figure 3. Immunocytochemically stained sections of rat brain using monoclonal antibody to erythroid GT viewed under Nomarski optics. The 2 high-magnification views illustrate localization of GT in capillaries of the cerebral cortex $(A)$ and of the corpus callosum $(B)$. Note that the reaction product appears to be restricted to the endothelium of brain capillaries, with no appreciable staining of the neuropil. Note the higher vascular density and the larger size of capillaries in gray matter than in white matter. Scale bar, $25 \mu \mathrm{m}$ for both $A$ and $B$.

thermore, the capillaries of the corpus callosum were smaller in diameter than those of the cerebral cortex (Fig. 3). Microvessel density also varied among the layers of the cerebral and cerebellar cortices. In the monkey and rat cerebral cortex, the highest and lowest densities of microvessels were in layers III-IV and I, respectively (Figs. $1 A, 2 A$ ). In the cerebellar cortex, capillary density was higher in the granular than in the molecular cell layer (Figs. $1 D, 2 D$ ). Certain brain-stem nuclei that are known for their dense vascularity, such as the nucleus locus coeruleus, were easily detected on immunostains for the GT (Fig. 1G). These results are consistent with common knowledge of variations in regional brain vascularity.

Our findings in the rat spinal cord were similar to those in the brain. All microvessels within and at the surface of the spinal cord stained for the GT (Fig. 4A,C). Again, we observed a distinctly higher vascular density in the spinal gray matter than in the white matter (Fig. 4A). The spinal meninges and the spinal ependyma did not stain for the GT. Microvessels of dorsal root ganglia (Fig. $4 B$ ) and spinal nerve roots (Fig. $4 C$ ) also stained.
In addition, the perineurium of nerve roots stained for GT (Fig. 4C).

Similar to spinal nerve roots, the rat sciatic nerve immunostained for the GT in both the endothelium of endoneurial vessels and the perineurium (Fig. $4 D$ ). There was no other staining above background in either the endoneurium or the epineurium and its blood vessels.

We studied 2 regions that are closely associated with the brain, but which are known to lack BBB characteristics: the pineal and the pituitary. There was no apparent staining above background for GT in the anterior or posterior lobes of the rat pituitary, nor in the rat pineal (Fig. $5 A, B$ ). However, in the monkey pineal, microvessels immunostained strongly for the GT (Fig. 5C).

Many tissues did not immunostain for the erythroid-type GT, neither in their vasculature nor in their parenchyma. These included the liver, the lung, fat tissue from several locations, skeletal muscle, and the heart. Examples of these tissues are shown in Figure 6. The perineurium of nerve fibers in skeletal muscles (Fig. 6D) and in the dermis of the skin (Fig. 6H) were stained. Only faint and diffuse immunostaining was observed in the jejunum of the small intestine, which was restricted to the serosa and the outer muscle layer (Fig. $6 F$ ), but there was no staining in the mucosa or submucosa. In the skin, there was faint but definite immunostaining for the GT in epidermal cells below the stratum corneum (Fig. $6 G$ ). In the kidney, no immunostaining was seen in the cortex in its blood vessels, glomeruli, or proximal and distal tubules (Fig. 6I). However, immunostaining was evident in the renal medulla and was notably restricted to the epithelium of the ascending tubules of Henle, which were identified by their location and by the intermediate thickness of their epithelium (Fig. $6 \mathrm{~J}$ ).

The testis was the only non-neural organ where immunostaining for GT was observed in its microvessels (Fig. 7A,B). However, unlike the brain and spinal cord, not all testicular microvessels were positively stained for the GT. A random count of 200 microvessels in sections of the rat testis that were doubleimmunostained for the GT and type IV collagen revealed that $84 \%$ of the microvessels were positive for both markers, and the rest lacked staining for the GT. No staining was observed within seminiferous tubules, neither in sperm precursors nor in Sertoli's cells. Also, no staining was observed in other cellular constituents of the interstitium (Fig. 7B). The ovaries (Fig. 7D) fallopian tubes, uterus, and prostate did not show positive immunostaining (results not shown).

\section{Discussion}

Recent studies suggest the existence of a family of GT proteins, which exhibit major amino acid residue sequence differences, in cell membranes of various tissues (Bell et al., 1990; Kasanicki and Pilch, 1990; Thorens et al., 1990). The tissue distribution of these GTs and the possible occurrence of cross-reactivity to them are not well known, and there is evidence that more than 1 GT may be present in the same tissue. However, in the experiments exemplified in Figure 6, tissues known to contain nonerythroid GTs did not immunostain with our antibodies (see above). The type of GT found in human erythrocytes is also abundant in brain microvessel membranes (Dick et al., 1984; Flier et al., 1987; Kalaria et al. 1988), but its precise localization and tissue distribution in the nervous system and in other organs is not yet known. In this study, we attempted to assess the tissue localization of the erythroid-type GT with particular attention to tissues that are known to have occluding 


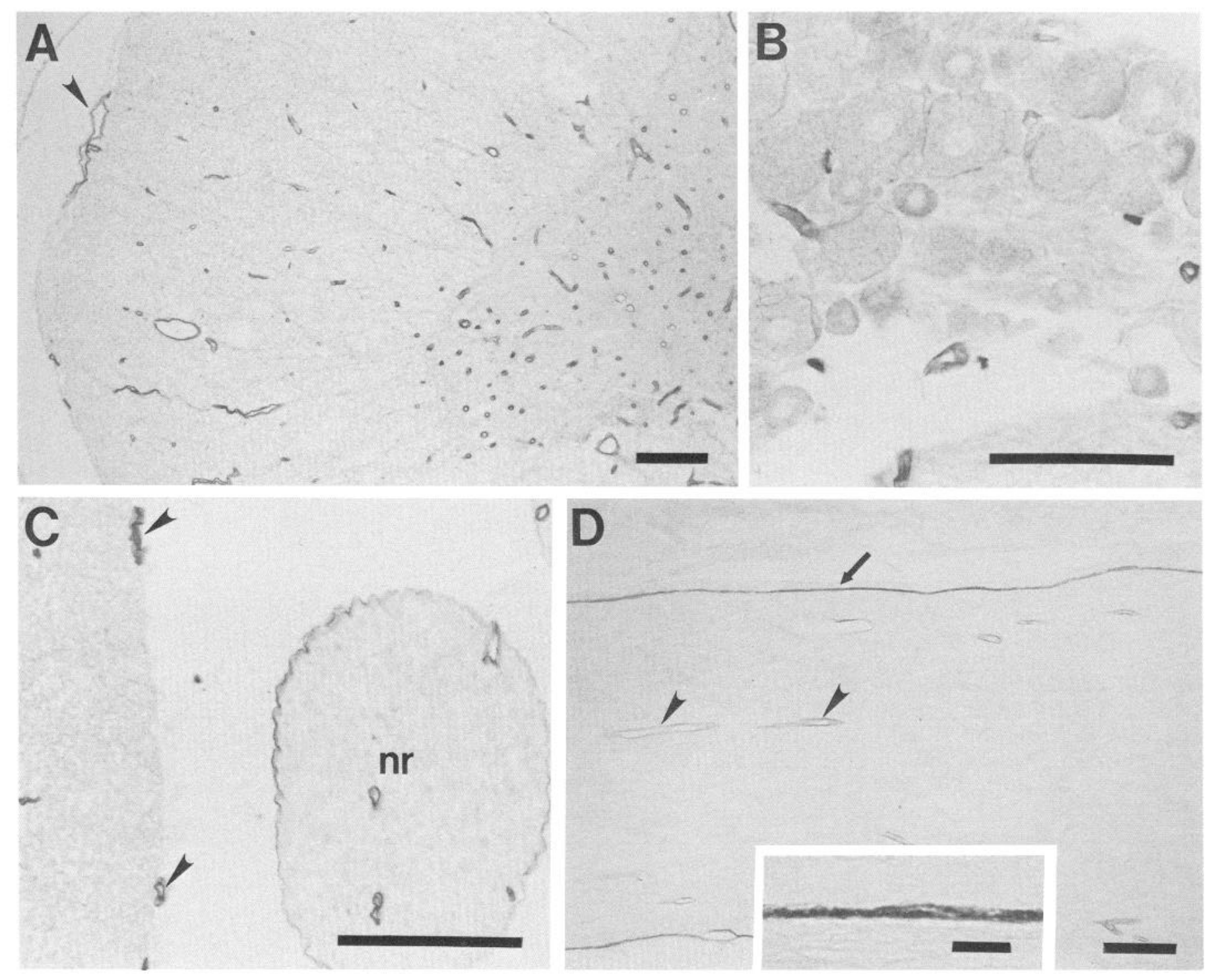

Figure 4. Immunocytochemical stains of tissue sections of rat spinal cord, dorsal root ganglion, and sciatic nerve. $A-C$ were taken from sections immunostained with the polyclonal antiserum to the erythroid GT. The monoclonal antibody to the erythoroid GT was used in $D$. $A$, A crosssection of the spinal cord showing immunostaining of microvessels, intraparenchymal and pial (arrowhead). Note the higher density of blood vessels in the inner gray matter of the cord than in the outer white matter. $B$, Immunostaining in microvessels of a dorsal root ganglion. $C$, A highermagnification view showing positive immunostaining of the pial vessels of the spinal cord (arrowhead) but no staining of the pia. There is also staining of endoneurial capillaries and the perineurium of a spinal nerve root $(n r)$. $D$, A longitudinal section of rat sciatic nerve showing immunostaining of endoneurial blood vessels (arrowheads) and of the perineurium (arrow). Blood vessels in the epineurium did not immunostain. The inset shows a high-power view of the perineurium. The inset in $D$ was photographed under Nomarski optics; all other portions of this figure were photographed under bright-field illumination. Scale bars: $A-D, 100 \mu \mathrm{m}$; inset in $D, 25 \mu \mathrm{m}$.

junctions. Our tissue distribution and cellular localization results are of interest because they allowed formulation of a testable hypothesis that may have major biological importance.

Our findings of intense immunostaining in brain microvessels of the rat and monkey with antibodies to the erythroid GT are consistent with the results of prior biochemical studies using ligand binding to isolated brain microvessels (Dick et al., 1984; Baldwin et al., 1985; Dick and Harik, 1986; Kasanicki et al., 1987; Kalaria et al., 1988), and with immunocytochemical studies on the human (Kalaria et al., 1988) and dog brain (Gerhart et al., 1989). Monkey and rat brain microvessels are highly enriched with the erythroid-type GT and stand out impressively against a low background in immunostains of brain tissue sec- tions using monoclonal or polyclonal GT antibodies (Figs. 1, 2). High-magnification views show that this immunostaining is mostly restricted to endothelial cells. This is also consistent with the ultrastructural studies of Gerhart et al. (1989) and our own results (J. W. Schmidley, S. I. Harik, R. N. Kalaria, and G. Perry, unpublished observations). The lack of definite immunostaining in most, but not all, of the neuropil may simply be a reflection of the major discrepancy between the high density of GT in brain microvessels and the low density in neurons and glia (Dick and Harik, 1986); it does not necessarily imply the absence of the erythroid GT in neurons and glia. Alternatively, the GT of neurons and glia may be immunologically distinct from that of human erythrocytes and brain microvessels. Against 

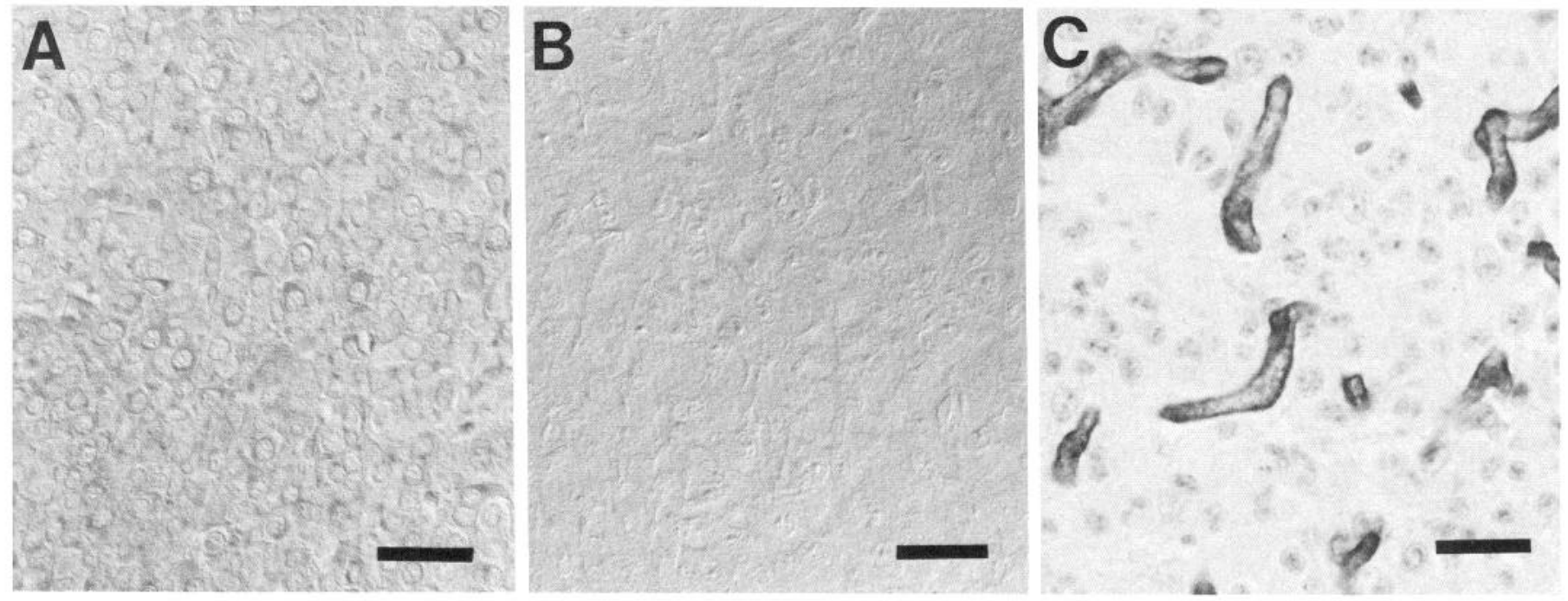

Figure 5. Immunocytochemical stains of section from rat pituitary $(A)$, rat pineal $(B)$, and monkey pineal $(C)$ using monoclonal antibody to erythroid GT. Immunostaining is evident only in capillaries of the monkey pineal $(C)$, but not in the rat pineal $(B)$ or pituitary $(A)$ glands. $A$ and $B$ were photographed under Nomarski optics, and $C$ was photographed under bright-field illumination after counterstaining with hematoxylin. Scale bars, $25 \mu \mathrm{m}$.

the latter possibility is the fact that tanycytes, which are specialized glia that exist in certain circumventricular regions of the brain, immunostain intensely with antibodies to the erythroid GT (Fig. 2E,G). These tanycytes are known to possess occluding, intercellular junctions that prevent the diffusion of horseradish peroxidase in the median eminence, where capillaries are known to lack BBB properties (Brightman and Reese, 1969).

Results from tissue sections that were double-immunostained with antiserum to collagen type IV, as a marker of the vascular basal lamina, and GT antibodies indicate that all brain microvessels, except those in circumventricular regions, immunostain for both markers. Thus, we believe that the erythroid GT is an excellent marker for brain microvessel endothelium. Even in the brains of Alzheimer's disease subjects, where the GT of brain microvessels is markedly reduced (Kalaria and Harik, 1989), the discordance between collagen type IV and the erythroid GT antibody reactivity in double-immunostained sections did not exceed $7 \%$ (Kawai et al., 1990). On the other hand, the endothelium of larger vessels, such as the basilar and anterior cerebral arteries, did not immunostain (Fig. $2 F$ ). This is compatible with the fact that larger blood vessels, unlike brain capillaries, do not play an important role in BBB transport.

All blood vessels of the brain and spinal cord that immunostained for the GT are known to possess occluding interendothelial junctions and are part of the BBB. As expected, en- doneurial microvessels of peripheral nerves stained for the GT, while perineurial vessels did not stain (Fig. $4 D$ ). Also, capillaries of the spinal roots and dorsal root ganglia immunostained for the GT, albeit not as strongly (Fig. 4B,C). This was not expected in view of the notion that these structures do not possess the same barrier characteristics as other neural capillaries (Shamboorov and Tchibukmakher, 1938; Waksman, 1961). Blood vessels of the choroid plexus of the rat and monkey, which do not have occluding junctions, did not immunostain (Figs. $1 H$, $2 H$ ). However, the choroid epithelium, which forms occluding junctions, was stained, mostly in its basal aspect. As in the choroid plexus, microvessels in circumventricular organs did not immunostain for the GT, as was evident from the rat pineal and pituitary (Fig. 5). Interestingly, in contrast to the rat pineal, the monkey pineal microvessels stained for the GT, similar to the finding in humans (Kalaria et al., 1988). We have no definitive knowledge of the barrier properties of pineal vessels in the human and monkey. However, if the high density of the erythroid GT is a marker of capillary endothelium with occluding junctions, we predict that pineal capillaries in the human and monkey, but not in the rat, are endowed with occluding junctions and have BBB properties.

In peripheral nerves, whether large (e.g., sciatic nerve; Fig. $4 B$ ) or small (e.g., nerve twigs in striated muscle and skin; Fig. $6 D, H$ ), proximal (e.g., spinal nerve roots; Fig. $4 C$ ) or distal, the most intense immunostaining for the erythroid GT was in the

Figure 6. Immunocytochemical stains in sections of rat tissues were performed using monoclonal antibody to erythroid GT. $A$, A section through the liver. The central vein $(\mathrm{cv})$ of a lobule is indicated. $B$, A section through the lung. $C$, A section through omental fat tissue. No staining is evident in either the blood vessels or parenchyma of these tissues. $D$, A section through skeletal muscles of the back showing immunostaining only in the perineuria of nerve fibers $(n)$. $E$, A section of cardiac muscle showing no immunostaining. $F$, A longitudinal section through the jejunum. There was minimal staining in the serosa and the underlying muscle layer. No staining was seen in the mucosa or submucosa. $G$, A section through the rat skin showing immunostaining of the epidermal cells below the striatum corneum. No staining is seen in blood vessels of the skin. $H$, A section through the dermis showing only positive immunostaining in the perineurium of a deep nerve fiber $(n)$. $I$, A section of the kidney cortex showing no immunostaining in the glomerulus $(\mathrm{gl})$, or in the proximal or distal tubules that surround it. $J$, A section through the medulla of the kidney showing positive staining in the ascending loop of Henle. No staining was seen in the proximal cortical tubules (pct), distal cortical tubules (dct), or in the thin descending loop of Henle $(d l h) . A-F, I$, and $J$ were photographed under differential interference contrast microscopy; $G$ and $H$ were photographed under bright-field illumination. Scale bars, $25 \mu \mathrm{m}$. 

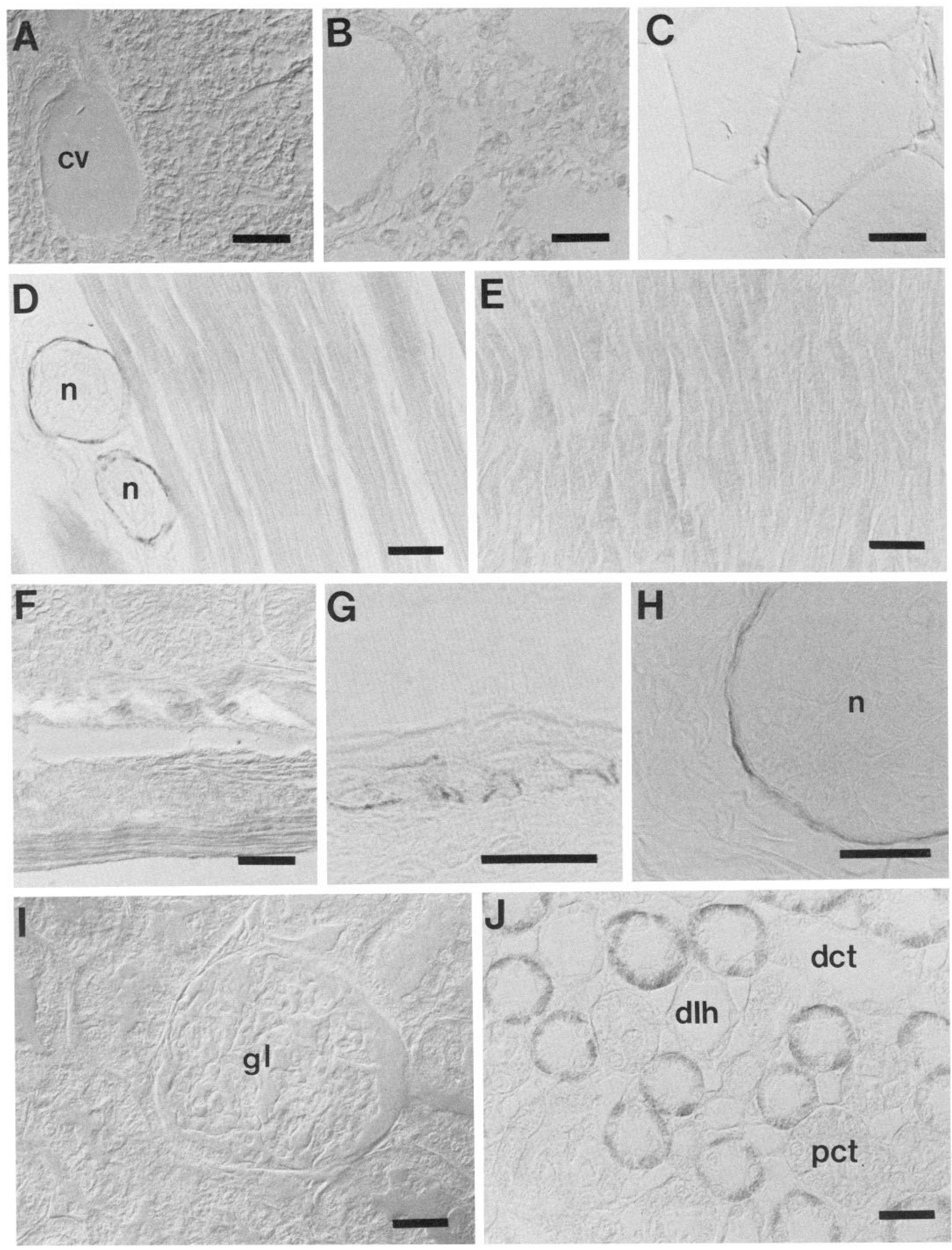


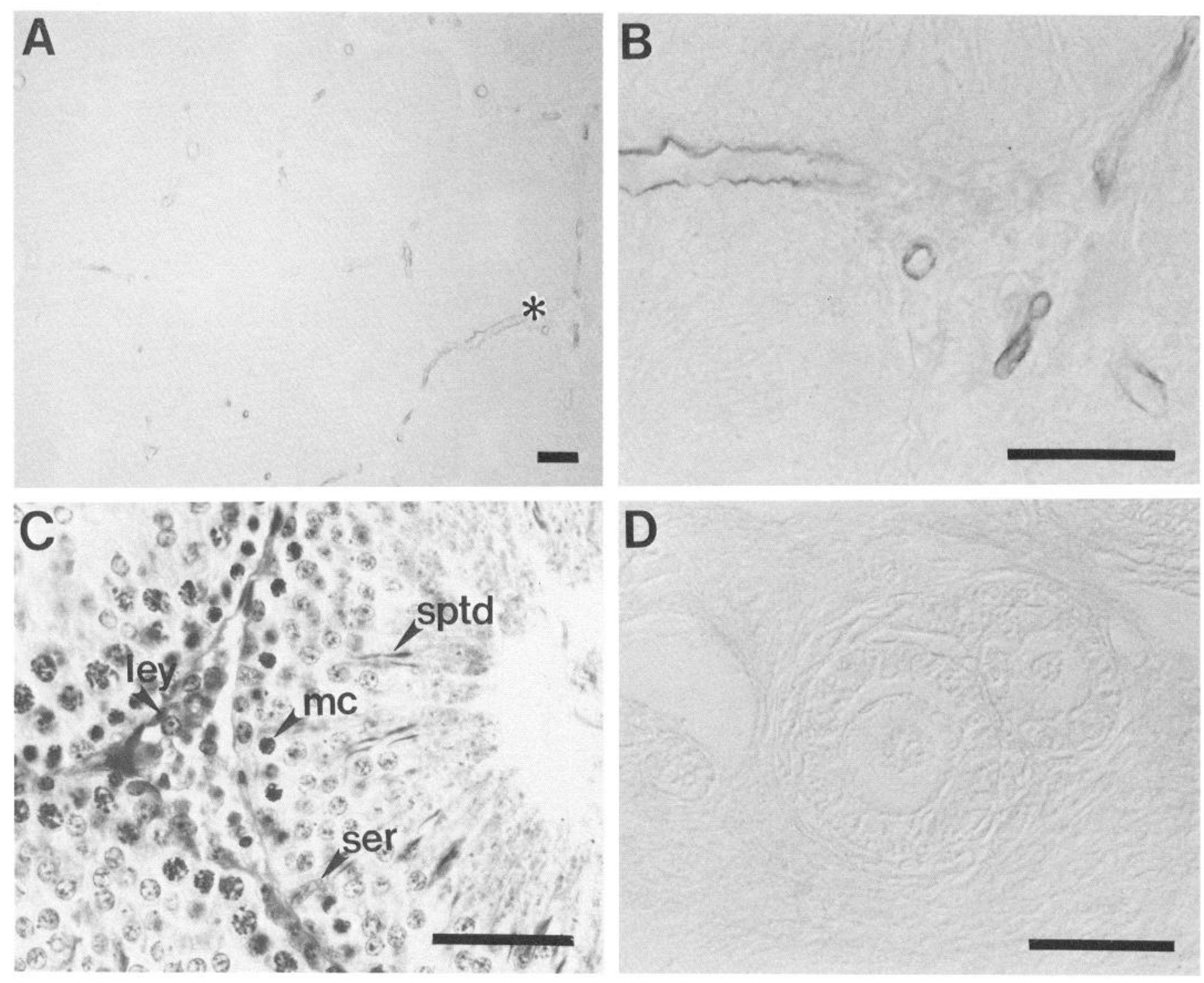

Figure 7. A, An immunostain of the rat testis using the monoclonal antibody to the erythroid GT. No staining is seen within the seminiferous tubules. However, most blood vessels in the interstitium stained well. This photograph was taken under bright-field illumination. $B$, A highmagnification view of the region in $A$ indicated by an asterisk, demonstrating the blood vessels in the testicular interstitium. This photograph was taken using Nomarski optics. $C$, An adjacent section of rat testis stained with hematoxylin and eosin to demonstrate the anatomy of the structures shown in $A$ and $B$. Spermatids (sptd), precursor undergoing miosis $(m c)$, Sertoli's cells (ser), and Leydig's cells (ley) are identified (bright-field microscopy). $D$, An immunostain of rat ovary using the monoclonal antibody to the GT. No immunostaining is seen in the follicles, stroma, or blood vessels (Nomarski microscopy). Scale bars, $50 \mu \mathrm{m}$.

perineurium. The perineurium consists of specialized fibroblasts that are known to form occluding intercellular junctions, and which are credited with an important role in preventing the diffusion of macromolecules from surrounding tissues into the nerve and in maintaining the integrity of the blood-nerve barrier (Olsson, 1968; Olsson and Reese, 1971).

Except for the CNS, PNS, and eye (Harik et al., 1990), the only organ where microvessels stained positively for the erythroid GT was the testis. Positive staining was identified only in the endothelium of the majority of testicular microvessels in the interstitium between seminiferous tubules (Fig. 7). Based on our findings, and consistent with our hypothesis that tissues with barrier properties have a high density of the GT, we suggest, contrary to the current view (Setchell et al., 1969; Setchell and
Waites, 1975), that the blood-testicular barrier is mainly a function of testicular capillaries. Ascribing the blood-testicular barrier to Sertoli's cell processes may be analogous to the now discarded idea that glial end-feet constitute the BBB.

Our findings in testicular microvessels were not unexpected. The concept of a blood-testis barrier is as old as that of the BBB and was derived by the same scientists who observed that, when dyes were injected into animals, most tissues were stained but not the brain or the testis (Bouffard, 1906; Goldmann, 1909). From a structural standpoint, testicular capillaries are not incompatible with a barrier function (Kormano, 1967, 1968). However, the barrier role of testicular capillaries was dealt a heavy blow by Setchell and collaborators (Setchell et al., 1969; Setchell and Waites, 1975), who showed that macromolecules, 
such as albumin, and water-soluble substances, such as L-glucose, readily appear in testicular lymph after systemic administration, though they do not gain entry into the rete testis fluid that drains seminiferous tubules (see review by Setchell and Waites, 1975). Their results were best explained by leakiness of testicular capillaries, which suggested that the blood-testis barrier existed at a more distal site. The finding of tight junctions in between Sertoli's cell processes was conveniently proposed as the barrier site.

We believe that these physiological findings may have other explanations. There is little question that some testis capillaries are permeable to L-glucose and albumin, if the existence of these substances in testicular lymph is accepted. However, we propose that only a minority of testis microvessels are leaky, similar to the minority of leaky brain vessels in circumventricular regions. Unlike the brain, however, we propose that, in the testis, such leaky capillaries are not restricted to distinct anatomical regions, but are widespread, and that the vast majority of testis capillaries have barrier properties. Our finding of intense immunostaining for GT in $84 \%$ of testicular microvessels is consistent with this suggestion, which can explain the physiological results of Setchell and his group, including the finding that the volume of distribution of L-glucose in the rat testis is $10-15 \%$ of that of D-methylglucose (Setchell and Waites, 1975). In fact, there already is ample anatomical ground for dividing testicular microvessels into 2 types (Setchell and Waites, 1975).

The questions concerning the factor(s) that underlie the formation of occluding junctions between endothelial or epithelial cells and the mechanism by which this is related to a high density of the erythroid-type GT in these cells remain unanswered. Glia or their influences are thought to play an important role in imparting barrier characteristics to capillary endothelium (Davson and Oldendorf, 1967; Stewart and Wiley, 1981; Janzer and Raff, 1987). If so, the same or similar events should be operating in the testis, which lacks glia. Also, it is not clear what causes the perineurial epithelium or the epithelium of the ascending loop of Henle to form occluding junctions and to contain a high density of the erythroid-type GT.

The findings we presented here support our starting hypothesis that a high density of the GT protein is a marker of many cells that possess occluding junctions. Such a hypothesis is not only reasonable, at least from a teleological standpoint, but may also be useful in identifying tissues with occluding junctions. An example of this is our findings of intense immunostaining of capillaries in the human and monkey pineal glands, suggesting that these vessels have barrier properties, which was not suspected before. The results in nonendothelial cells also support our hypothesis and may be even more provoking. The perineurium, tanycytes at the base of the third ventricle, and the epidermis all possess tight junctions and immunostained intensely for the erythroid GT. However, other cells that have occluding junctions did not stain for the erythroid GT. Prominent among the latter are the mucosal epithelium of the small intestine and much of the tubular epithelium of the kidney. Our explanation is that the GT in these tissues is different from the erythroidtype GT. Glucose transport in the intestine and in the proximal tubules of the kidney is known to be an energy-dependent and concentrative process, and it is therefore likely that their GT is of the sodium-dependent, concentrative type (Semenza et al., 1984; Hediger et al., 1987; Baly and Horuk, 1988; Silverman, 1989); but why, then, should the ascending loop of Henle, of all the kidney epithelia, possess the erythroid GT? We suspect that the answer may be related to the marked differences in $\mathrm{Na}^{+}$ concentration across the cpithclium of the ascending loop of Henle, which could make the $\mathrm{Na}^{+}$-dependent glucose transport difficult.

\section{References}

Andersson L, Lundahl P (1988) C-terminal-specific monoclonal antibodies against the human red cell glucose transporter. J Biol Chem 263:11414-11420.

Baldwin SA, Cairns MT, Gardiner RM, Ruggier R (1985) A D-glucosesensitive cytochalasin $B$ binding component of cerebral microvessels. J Neurochem 45:650-652.

Baly DL, Horuk R (1988) The biology and biochemistry of the glucose transporter. Biochim Biophys Acta 947:571-590.

Bell GI, Kavano T, Buse JB, Burant CF, Takeda J, Lin D, Fukumoto H, Seino S (1990) Molecular biology of mammalian glucose transporters. Diabetes Care 13:198-208.

Bouffard G (1906) Injection des couleurs de benzidine aux animaux normaux. Ann Immunol (Paris) 20:539-546.

Brightman MW, Reese TS (1969) Junctions between intimately apposed cell membranes in the vertebrate brain. J Cell Biol 40:648677.

Crone C (1961) Om diffusionen af nogle organiske non-elektrolyter fra blod til hjernevaev. PhD thesis, University of Copenhagen.

Crone C (1963) The permeability of capillaries in various organs as determined by use of the "indicator diffusion" method. Acta Physiol Scan 58:292-305.

Crone C (1965) Facilitated transfer of glucose from blood into brain tissue. J Physiol (Lond) 181:103-113.

Davson H, Oldendorf WH (1967) Transport in the central nervous system. Proc R Soc Med 60:326-328.

Dick APK, Harik SI (1986) Distribution of the glucose transporter in the mammalian brain. J Neurochem 46:1406-1411.

Dick APK, Harik SI, Klip A, Walker DM (1984) Identification and characterization of the glucose transporter of the blood-brain barrier by cytochalasin B binding and immunological reactivity. Proc Natl Acad Sci USA 81:7233-7237.

Flier JS, Mueckler M, McCall AL, Lodish HF (1987) Distribution of glucose transporter messenger RNA transcripts in tissues of rat and man. J Clin Invest 79:657-661.

Frochner SC, Davies A, Baldwin SA, Lienhard GE (1988) The bloodnerve barrier is rich in glucose transporter. J Neurocytol 17:173-178.

Gerhart DZ, LeVasseur RJ, Broderius MA, Drewes LR (1989) Glucose transporter localization in brain using light and electron immunocytochemistry. J Neurosci Res 22:464-472.

Goldmann EE (1909) Die aussere und innere sekretion des gesunden und kranken Organismus im Lichte der "vitalen Farbung." Beitr Klin Chirug 64:192-265.

Harik SI, Whitney P, Perry G, Kalaria, RN, Andersson L, Lundahl P (1989) Tissue distribution and cellular localization of the glucose transporter by immunocytochemistry. Neurology [Suppl 1] 39:371.

Harik SI, Kalaria RN, Whitney PM, Andersson L, Lundahl P, Ledbetter S, Perry G (1990) Glucose transporters are abundant in cells with "occluding" junctions at the blood-eye barriers. Proc Natl Acad Sci USA $87: 4261-4264$.

Haspel HC, Birnbaum MJ, Wilk EW, Rosen OM (1985) Biosynthetic precursors and in vitro translation products of the glucose transporter of human hepatocarcinoma cells, human fibroblasts, and murine preadipocytes. J Biol Chem 260:7219-7225.

Haspel HC, Rosenfeld MG, Rosen OM (1988) Characterization of antisera to a synthetic carboxyl-terminal peptide of the glucose transporter protein. J Biol Chem 263:398-403.

Hediger MA, Coady MJ, Ikeda TS, Wright EM (1987) Expression cloning and cDNA sequencing of $\mathrm{Na}^{+} /$glucose co-transporter. Nature 330:379-381.

Janzer RC, Raff MC (1987) Astrocytes induce blood-brain barrier properties in endothelial cells. Nature 325:253-257.

Kalaria RN, Harik SI (1989) Reduced glucose transporter at the bloodbrain barrier and in cerebral cortex in Alzheimer's disease. J Neurochem 53:1083-1088

Kalaria RN, Gravina SA, Schmidley JW, Perry G, Harik SI (1988) The glucose transporter of the human brain and blood-brain barrier. Ann Neurol 24:757-764.

Kalaria RN, Usiak M, Golde T, Younkin L, Harik SI (1989) Expres- 
sion of the glucose transporter mRNA in rat and human cerebral cortex demonstrated by in situ hybridization. Soc Neurosci Abst 15: 820.

Kasanicki MA, Pilch PE (1990) Regulation of glucose-transporter function. Diabetes Care 13:219-227.

Kasanicki MA, Cairns MT, Davies A, Gardiner RM, Baldwin SA (1987) Identification and characterization of the glucose-transport protein of the bovine blood-brain barrier. Biochem J 247:101-108.

Kawai M, Kalaria RN, Harik SI, Perry G (1990) The relationship of amyloid plaques to cerebral capillaries in Alzheimer's disease. Am J Pathol, in press.

Kormano M (1967) Dye permeability and alkaline phosphatase activity of testicular capillaries in the postnatal rat. Histochemie 9:327338.

Kormano M (1968) Penetration of intravenous trypan blue into the rat testis and epididymis. Acta Histochem 30:133-136.

Olsson Y (1968) Topographical differences in the vascular permeability of the peripheral nervous system. Acta Neuropathol 10:26-33.

Olsson Y, Reese TS (1971) Permeability of vasa nervorum and perineurium in mouse sciatic nerve studied by fluorescence and electron microscopy. J Neuropathol Exp Neurol 30:105-119.

Semenza G, Kessler M, Hosang M, Weber I, Schmidt U (1984) Biochemistry of the $\mathrm{Na}^{+}, \mathrm{D}$-glucose cotransporter of the small-intestinal brush-border membrane. Biochim Biophys Acta 779:343-379.
Setchell BP, Waites GMH (1975) The blood-testis barrier. In: Handbook of physiology, Sec 7, Vol V (Hamilton VW, Greep RO, eds), pp 143-172. Washington: American Physiological Society.

Setchell BP, Voglmayr JK, Waites GMH (1969) A blood-testis barrier restricting passage from blood in to rete testis fluid but not into lymph. J Physiol (Lond) 200:73-85.

Shamboorov DA, Tchibukmakher NB (1938) Contribution to the problem of the peripheral nervous barrier. Acta Med Scan 47:175206.

Silverman M (1989) Molecular biology of the $\mathrm{Na}^{+}-\mathrm{D}-$ glucose cotransporter. Hosp Pract 24:180-204.

Sternberger LA (1986) Immunocytochemistry. New York: Wiley.

Stewart PA, Wiley MJ (1981) Developing nervous tissue induces formation of blood-brain barrier characteristics in invading endothclial cells: a study using quail-chick transplantation chimeras. Dev Biol 84:183-192.

Thorens B, Charron MJ, Lodish HF (1990) Molecular physiology of glucose transporters. Diabetes Care 13:209-218.

Waksman BH (1961) Experimental study of diphtheric polyneuritis in the rabbit and guinea pig. III. The blood-nerve barrier in the rabbit. J Neuropathol Exp Neurol 20:35-77.

Weiler-Güttler H, Zinke H, Möckel B, Frey A, Gassen HG (1989) cDNA cloning and sequence analysis of the glucose transporter from porcine blood-brain barrier. Biol Chem Hoppe-Zeyler 370:467-473. 